\title{
Striktes Schema zur Therapie bei rheumatoider Arthritis
}

Schneller Therapie-Start, schnell in die Remission lautet das Ziel bei rheumatoider Arthritis. Kommen Biologika zum Einsatz, ist zu beachten, dass die Serumspiegel interindividuell stark schwanken. Eine Umstellung auf Biosimilars scheint kaum Probleme zu bereiten.

Das Therapie-Ziel bei Patienten mit rheumatoider Arthritis (RA) ist weiterhin die Remission und deren Erhalt. „Dies wird erreicht durch einen frühestmöglichen Therapiebeginn und ein striktes Treat-to-Target-Schema", sagte Prof. Dr. Klaus Krüger, Praxiszentrum St. Bonifatius, München. Begonnen wird mit Methotrexat und $10-30 \mathrm{mg}$ Prednisolon. Zunächst nach sechs bis zwölf, dann alle zwölf Wochen wird die Krankheitsaktivität kontrolliert. Diese sollte in Woche zwölf um mindestens $50 \%$ zurückgegangen sein, nach 26 Wochen sollte der Patient in Remission sein. Wird dieses Ziel nicht erreicht, erfolgt eine Therapie-Eskalation durch Kombination von Basistherapeutika (DMARD). Sind die Prognosefaktoren ungünstig und die Krankheitsaktivität hoch, wird ein Biologikum oder ein JAK-Hemmer eingesetzt.

„Das Glukokortikoid ist in der Starttherapie unverzichtbar, es sollte spätestens nach acht Wochen den Low-DoseBereich erreicht haben", so Krüger beim RheumaUpdate in Wiesbaden. In der Realität werden allerdings 40 bis $50 \%$ der Patienten dauerhaft mit einem Glucokortikoid behandelt. Und nur 3\% erhalten im ersten Jahr ein Biologikum. Der immer wieder diskutierte TherapieAbbau sei allenfalls denkbar bei seit mindestens sechs Monaten bestehender stabiler Remission, sagte Krüger. Ihm müsse eine gemeinsame Entscheidung von Arzt und Patient zugrunde liegen.

Beurteilt wird die Krankheitsaktivität bildeten die klinische Situation besser $a b$, seien weniger fehlerbehaftet und zudem zeitsparend, so Krüger. (Scores unter www.rheumakit.com)

Krüger wies darauf hin, dass die Serumspiegel des TNFa-Blockers Adalimumab eine große interindividuelle Variabilität aufweisen. „Das Biologikum kann sowohl über-als auch unterdosiert werden", warnte der Rheumatologe. Entwickelten Patienten keine Serumspiegel, mache es keinen Sinn, mit Adalimumab weiter zu therapieren. Bei hohen Serumspiegeln wiederum könne eine Verlängerung der Injektionsintervalle erfolgen. Das wurde in einer offenen Studie mit RA-Patienten und Adalimumab-Serumspiegeln über $8 \mu \mathrm{g} / \mathrm{ml}$ gezeigt. Ein Teil erhielt das Biologikum in üblicher Frequenz, beim anderen Teil wurde die Frequenz auf drei Wochen verlängert [1]. In der Prolongationsgruppe ging der DAS28 um 0,14 Punkte zurück, in der Standardgruppe nahm er um 0,3 Punkte zu.„Die Adalimumab-Spiegel zu messen, sollten wir in unser Repertoire aufnehmen", empfahl Krüger.

Bei den Biologika feiern die Biosimilars einen Siegeszug. Ihr Anteil liege bei Etanercept bei rund $52 \%$ und bei Infliximab bei rund $65 \%$. Bei Adalimumab habe sich der Biosimilar-Anteil auf $42 \%$ entwickelt - und das nur binnen drei Monaten. Es gebe regionale Unterschiede bei der Biosimilar-Quote. Das Einhalten der Quote schütze nicht vor Einzelfallprüfungen. Einige Kassen gingen immer vor, werde ein Original verordnet.„Bei bestehenden Rabattverträgen gibt es keine Unwirtschaftlichkeit", betonte Krüger. Die Rabattverträge zu kennen, sei daher nützlich. Letztlich komme man nicht umhin, umzustellen, auch wenn dies dem ,Shared-DecisionMaking" widerspreche.

Der Wechsel auf ein Biosimilar kann ohne große Probleme verlaufen, hat eine Auswertung der Daten des dänischen Biologika-Registers DANBIO gezeigt. Hier wechselten 1621 von 20.161 Patienten mit stabiler Krankheitsaktivität von RA, Psoriasis-Arthritis oder axialer Spondyloarthritis vom Etanercept-Original auf ein Biosimilar [2]. Nach einem Jahr lag die Retentionsrate bei den Switchern bei $83 \%$, bei den Non-Switchern bei $77 \%$. Es wechselten $7 \%$ zurück auf das Original, $6 \%$ wechselten das Biologikum - die Gründe seien überwiegend subjektiver Art gewesen, so Krüger.

Ein striktes Alkoholverbot sei unter Methotrexat-Therapie nicht nötig, sondern möglicherweise sogar kontraproduktiv. Krüger zitierte eine Studie, in der das Ansprechen auf eine MTX-Therapie untersucht wurde [3]: Rauchen erhöhte das Risiko für schlechtes Ansprechen (OR 3,0) ebenso wie ein hoher DAS28 zu Beginn (OR 2,1). Mäßiger Alkoholkonsum hingegen begünstigte das Ansprechen (OR 0,35).,_Das wird unsere Patienten freuen."

\section{Literatur \\ 1. (2018) Ann Rheum Dis. $77: 484$ \\ 2. (2019) Ann Rheum Dis. 78:192 \\ 3. (2018) Ann Rheum Dis. 77:1261}

rheuma plus $2019 \cdot 18: 96$ https://doi.org/10.1007/s12688-0190259-6

(c) Springer-Verlag GmbH Austria, ein Teil von Springer Nature 2019 